\title{
Black Holes and Core Expansion in Massive Star Clusters
}

\author{
A. D. Mackey ${ }^{1}$, M. I. Wilkinson ${ }^{2}$, M. B. Davies $^{3}$ and G. F. Gilmore ${ }^{4}$ \\ ${ }^{1}$ Institute for Astronomy, University of Edinburgh, Royal Observatory, Blackford Hill, \\ Edinburgh, EH9 3HJ, UK; email: dmy@roe.ac.uk \\ ${ }^{2}$ Department of Physics \& Astronomy, University of Leicester, University Road, \\ Leicester, LE1 7RH, UK \\ ${ }^{3}$ Lund Observatory, Box 43, SE-221 00 Lund, Sweden \\ ${ }^{4}$ Institute of Astronomy, University of Cambridge, Madingley Road, \\ Cambridge, CB3 0HA, UK
}

\begin{abstract}
Massive star clusters in the Magellanic Clouds are observed to follow a striking trend in size with age - older clusters exhibit a much greater spread in core radius than do younger clusters, which are generally compact. We present results from realistic $N$-body modelling of massive star clusters, aimed at investigating a dynamical origin for the radius-age trend. We find that stellar-mass black holes, formed as remnants of the most massive stars in a cluster, can constitute a dynamically important population. If retained, these objects rapidly form a dense core where interactions are common, resulting in the scattering of black holes into the cluster halo, and the ejection of black holes from the cluster. These processes heat the stellar component, resulting in prolonged core expansion of a magnitude matching the observations. Core expansion at early times does not result from the action of black holes, but can be reproduced by the effects of rapid mass-loss due to stellar evolution in a primordially mass segregated cluster.
\end{abstract}

Keywords. Stellar dynamics, N-body simulations, galaxies: star clusters, Magellanic Clouds

\section{Introduction and Numerical Setup}

For observational studies of star cluster evolution, the Galactic globular clusters, while close, are not ideal because they are exclusively ancient objects $\left(\tau \geqslant 10^{10} \mathrm{yr}\right)$. We can therefore accurately assess the end-points of their evolution, but must infer the complete long-term development which brought them to these observed states. To directly observe cluster evolution, we must switch our attention to the Magellanic Clouds (LMC/SMC), which both possess extensive systems of star clusters with masses comparable to the Galactic globulars, but crucially of all ages: $10^{6} \leqslant \tau \leqslant 10^{10}$ yr. These systems are of fundamental importance because they are the nearest places we can observe snapshots of all phases of massive star cluster development.

Elson, Freeman \& Lauer (1989) discovered a striking relationship between core radius $\left(r_{c}\right)$ and age for LMC clusters - the observed spread in $r_{c}$ increases dramatically with increasing age. More recently, Mackey \& Gilmore (2003a,b) used Hubble Space Telescope (HST) WFPC2 imaging of 63 massive Magellanic Cloud clusters to more clearly demonstrate the radius-age trend in the LMC and show, for the first time, that an indistinguishable radius-age trend also exists in the SMC. An additional 46 objects have since been observed with HST/ACS (Program \#9891) to improve sampling of the radius-age plane. Structural measurements for all 107 clusters may be seen in Fig. 1.

The observed radius-age relationship provides strong evidence that our understanding of massive cluster evolution is incomplete, since standard quasi-equilibrium models do not predict long-term large-scale core expansion. Discerning the origin of the radius-age trend 
is therefore of considerable importance. Various observational evidence (summarised in Mackey et al. 2007b in preparation ) suggests that the radius-age relation is driven by internal cluster processes, with any external or tidal effects second order (see also Wilkinson et al. 2003). In this contribution, we report on the results of direct, realistic $N$-body simulations designed to investigate an internal dynamical origin for the radius-age trend.

We have used the NBODY 4 code (Aarseth 2003) in combination with a 32-chip GRAPE6 special-purpose computer (Makino et al. 2003) to simulate massive star clusters over a Hubble time of evolution. We generate initial conditions with properties (masses, structures, densities, etc) as similar as possible to those observed for the youngest Magellanic Cloud clusters. Full details are provided in Mackey et al. (2007a) and Mackey et al. (2007b in preparation); here we simply note that our simulated clusters start with projected radial density profiles described by Elson, Fall \& Freeman (1987; hereafter EFF) models with a power-law fall-off $\gamma=3$. Initial stellar velocities are drawn from a Maxwellian distribution, where the velocity dispersion is calculated using the Jeans equations assuming an isotropic velocity distribution. We select the IMF of Kroupa (2001), with a stellar mass range $0.1-100 \mathrm{M}_{\odot}$, so that with $N \sim 10^{5}$ particles our cluster models possess total masses $\log M_{\text {tot }} \sim 4.75$, consistent with massive young Magellanic Cloud objects. The models move on circular orbits of radius $6 \mathrm{kpc}$ about a point-mass LMC with $M_{\mathrm{g}}=9 \times 10^{9} \mathrm{M}_{\odot}$.

For the models presented in this contribution, we are most interested in examining the dynamical effects of a retained population of stellar-mass black holes (BHs). We modified NBODY4 to control the production of BHs in supernova explosions. In the simulations described here, all stars initially above $20 \mathrm{M}_{\odot}$ produce $\mathrm{BHs}$, with masses uniformly distributed in the range $8 \leqslant m_{\mathrm{BH}} \leqslant 12 \mathrm{M}_{\odot}$. Our adopted IMF and total $N$ lead to the formation of $198 \mathrm{BHs}$ in all clusters. Natal BH velocity kicks are either much larger than the cluster escape velocity $\left(\mathrm{BH}\right.$ retention fraction $\left.f_{\mathrm{BH}}=0\right)$, or zero $\left(f_{\mathrm{BH}}=1\right)$.

Many young LMC and SMC clusters exhibit some degree of mass segregation. In order to include the effects of this in our models, we developed a method to generate clusters with primordial mass segregation in a self-consistent fashion; again, full details are in Mackey et al. (2007b in preparation). Here we consider models either with no primordial mass segregation, or a strong degree of primordial mass segregation chosen to match that observed in young Magellanic Cloud objects such as R136, NGC 330, 1805, and 1818.

To obtain structural measurements consistent with those for real clusters, we simulate observations of our $N$-body models. That is, we mimic the reduction procedures from which the HST $r_{c}$ measurements were derived. We first convert the luminosity and effective temperature of each $N$-body star to magnitude and colour. Next, we impose bright and faint detection limits commensurate with the original observations, along with fieldof-view limits appropriate for WFPC2 and ACS. We use the remaining stars to construct a projected radial profile and finally fit an EFF model to derive $r_{c}$.

\section{Results}

The parameter space of interest is spanned by non mass segregated clusters and those with significant primordial mass segregation. For each of these, we consider evolution with $\mathrm{BH}$ retention fractions $f_{\mathrm{BH}}=0$ and 1 . These four runs define the extremities of the parameter space, and hence are expected to cover the limits of cluster behaviour.

We first consider Runs 1 and 2, which have no primordial mass segregation, and $f_{\mathrm{BH}}=$ 0 and 1, respectively. Their evolution is visible in the left panel of Fig. 1. Run 1 behaves exactly as expected for a classical massive star cluster. There is an early mass-loss phase $(\tau \leqslant 100 \mathrm{Myr})$ due to the evolution of the most massive cluster stars. BHs are formed in 

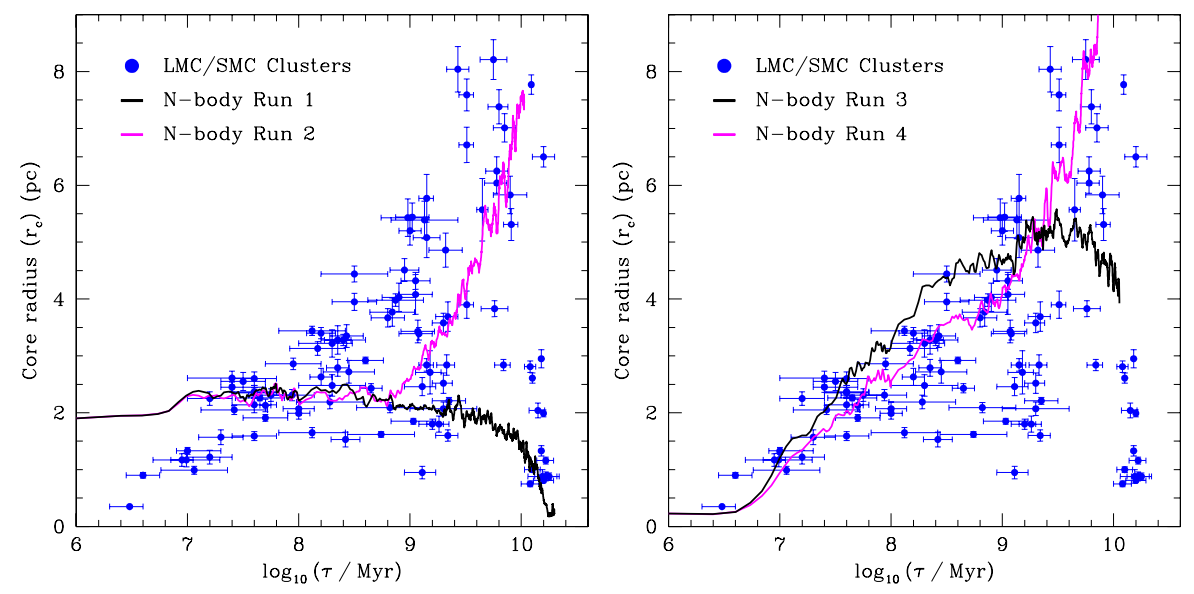

Figure 1. Left: Core radius evolution of Runs 1 and 2 (lower and upper lines, respectively). Right: Core radius evolution of Runs 3 and 4 (lower and upper lines, respectively).

supernova explosions between 3.5-10 Myr; however, all receive large velocity kicks and escape. The early mass-loss is not reflected in the evolution of $r_{c}$, presumably because it is evenly distributed throughout the cluster. Subsequently, the general progression is a slow contraction as two-body relaxation proceeds and mass segregation sets in. The cluster core collapses near the end of the simulation.

Run 2 evolves similarly to a point, but, in striking contrast to Run 1, subsequently undergoes dramatic and long-term core expansion. The only difference between this model and Run 1 is that $f_{\mathrm{BH}}=1$ - i.e., $198 \mathrm{BHs}$ are retained. Once early stellar evolution is complete, the BHs are more massive than all other cluster members (of mean mass $\left.m_{*} \approx 0.5 \mathrm{M}_{\odot}\right)$ and hence sink to the core on a time-scale of $\sim\left(m_{*} / m_{\mathrm{BH}}\right) t_{r h} \approx 100 \mathrm{Myr}$. By $200 \mathrm{Myr}$, the mass density of BHs at the cluster centre is similar to that of the stars; by 400 Myr it is about three times larger. Soon after, the central BH subsystem becomes unstable against further contraction and decouples from the stellar core in a runaway collapse. At $490 \mathrm{Myr}$, the central density of the BH subsystem is $\sim 80$ times that of the stars. This is sufficient for the creation of stable $\mathrm{BH}$ binaries in three-body interactions the first is formed at $\sim 510 \mathrm{Myr}$, and by $800 \mathrm{Myr}$ there are four.

Until this phase the evolution of Run 2 is observationally identical to that of Run 1 . Neither BH retention, nor the subsequent formation of a central BH subsystem leads to differential evolution of $r_{c}$. Once formed, binary BHs undergo superelastic collisions with other BHs in the core. The binaries become "harder", and the released binding energy is carried off by the interacting BHs. This leads to BHs being scattered outside $r_{c}$, often into the cluster halo, as well as to BHs being ejected from the cluster. Eventually a BH binary is sufficiently hard that the recoil velocity imparted to it during a collision is larger than the cluster escape velocity, and the binary is ejected. A BH scattered into the halo gradually sinks back into the centre via dynamical friction, thus transferring its newly-gained energy to the stellar component of the cluster. Most is deposited within $r_{c}$, where the stellar density is greatest. Ejected BHs also transfer energy to the cluster, since a mass $m$ escaping from a cluster potential well of depth $|\Phi|$ does work $m|\Phi|$ on the cluster. This mechanism is particularly effective in heating the stellar core, since BHs are ejected from the very centre of the cluster, and the energy contributed to each part of the cluster is proportional to the contribution which that part makes to the central potential. Together these two processes result in significant prolonged core expansion, starting at 
$\tau \approx 650$ Myr. The size of $r_{c}$ behaves roughly as $\log \tau$, consistent with the upper envelope of the observed cluster distribution. However, in this model the expansion begins too late for the evolution to trace the upper envelope; rather, it runs parallel. Merritt et al. (2004) observed expansion due to similar processes in their simplified $N$-body models.

The number of stable BH binaries in the system peaks at 5 at $\tau \approx 890 \mathrm{Myr}$, after which there are $0-5$ at any given time. Single and binary BHs are continually ejected; however, empirically, both escape rates decrease with time such that $d^{2} N_{\mathrm{e}} / d \tau^{2} \propto-1 / \tau$. This is a result of the density of the central BH subsystem gradually decreasing with time due to the expansion of the cluster and the ejection of BHs. This in turn leads to a decreasing $\mathrm{BH}-\mathrm{BH}$ encounter rate. By $\tau \approx 10 \mathrm{Gyr}, 65$ single BHs and 2 binary BHs remain in the model. This is at odds with early studies (e.g., Kulkarni et al. 1993; Sigurdsson \& Hernquist 1993) which predicted depletion of retained $\mathrm{BH}$ populations on timescales much less than cluster lifetimes. The decreasing $\mathrm{BH}$ encounter rate seen in our models prolongs the life of the BH subsystem for much longer than previously appreciated.

The velocity and mass distributions of stellar escapers are indistinguishable for Runs 1 and 2, implying that both lose stars solely due to relaxation processes. In Run 2 it is clear that stars interact closely with BH binaries only very rarely. Heating of the stellar component via such close interactions is hence negligible - the hardening of $\mathrm{BH}$ binaries is driven solely through interactions with other BHs.

Next, consider the evolution of Runs 3 and 4, which are primordially mass segregated versions of Runs 1 and 2, respectively (Fig. 1, right panel). In these models, early massloss due to stellar evolution is highly centrally concentrated - hence the amount of heating per unit mass lost is maximised, leading to dramatic early core expansion. Run 3 traces the observed upper envelope of clusters until several hundred Myr. Run 4 retains its BHs and hence loses less mass than Run 3 - this is reflected in its smaller $r_{c}$. After the early mass-loss phase is complete, core expansion stalls in both runs. Two-body relaxation gradually takes over in Run 3 , leading to a slow contraction in $r_{c}$. At $\tau=1$ Gyr, $t_{r h} \approx 4$ Gyr; hence this cluster is not near core collapse by the end of the simulation.

In Run 4, the $\mathrm{BH}$ population evolves similarly to that in Run 2. One might naively expect the earlier development of a compact BH subsystem in Run 4, because the BHs are already located in the core due to the primordial mass segregation. However, the centrally concentrated mass-loss acts against the accumulation of a dense BH core, and the first binary $\mathrm{BH}$ does not form until $570 \mathrm{Myr}$, a similar time to the non mass segregated model. The BH subsystem evolves more slowly than that in Run 2 - by $\tau=10$ Gyr, there are still 95 single BHs and 2 binary BHs remaining in the cluster. As in Run 2, the evolution of the BH subsystem leads to expansion of $r_{c}$. This begins at $\tau \approx 800 \mathrm{Myr}$ and continues for the remainder of the simulation. As previously, $r_{c}$ behaves roughly as $\log \tau$ during this phase. By $\tau \approx 10 \mathrm{Gyr}$, Run 4 has $r_{c} \sim 11 \mathrm{pc}$, comparable to that observed for the most extended old Magellanic Cloud clusters (e.g., Reticulum).

\section{Discussion}

Our four simulations cover the observed cluster distribution in radius-age space, thereby defining a dynamical origin for the radius-age trend. At ages less than a few hundred Myr, cluster cores expand due to centrally concentrated mass-loss from stellar evolution. At later times, expansion is induced via heating due to a $\mathrm{BH}$ population. Although we have assumed $f_{\mathrm{BH}}=1$, full retention is not necessary for cluster expansion. $\mathrm{BH}$ kicks of order $10 \leqslant v_{\text {kick }} \leqslant 20 \mathrm{kms}^{-1}$ result in $f_{\mathrm{BH}} \sim 0.5$ in our models; evolution in such systems is intermediate between that of Runs 1 and 2, or 3 and 4 (Mackey et al. 2007b in preparation). 
Our models require variations in $\mathrm{BH}$ population size between otherwise similar clusters. This is discussed fully by Mackey et al. 2007b in preparation; here, we simply note a few possibilities. First, the fraction of BH-forming stars in a cluster is small, so there will be sampling-noise variations between clusters. Further, any dispersion in stellar rotation may introduce mass-loss variations and further dispersion in BH numbers. Metallicity is also likely to be a key factor. Natal BH kicks are poorly constrained at present - typical estimates are $0 \leqslant v_{\text {kick }} \leqslant 200 \mathrm{~km} \mathrm{~s}^{-1}$. Stellar binarity may therefore play a significant role in retaining cluster $\mathrm{BHs}$, as will the initial cluster mass and degree of primordial segregation.

Galactic globular clusters are typically an order of magnitude more massive than our $N$-body models. However, we expect the evolution described above to scale to such objects. The mass fraction of BHs formed in a cluster is dependent only on the IMF and minimum progenitor mass, neither of which should change with $M_{\text {tot }}$, while a larger $M_{\text {tot }}$ implies a larger $f_{\mathrm{BH}}$ since it is easier to retain newly-formed BHs. The densities in our models are consistent with those observed for globular clusters; hence we expect the same processes to operate on similar time-scales, although BHs are likely to be more difficult to eject in more massive clusters - increasing the potential of each $\mathrm{BH}$ to heat the cluster via additional scattering-sinking cycles.

Core expansion due to early mass-loss and prolonged BH heating has strong implications for the observed properties of globular clusters as well as their survivability. Extended clusters are significantly more susceptible to tidal disruption, so it is important to account for expansion effects in studies of the evolution of the globular cluster mass function, for example. Core expansion due to BHs may offer a viable explanation for the origin of the luminous, unusually extended globular clusters found in M31, which are $>10$ Gyr old metal-poor objects (Mackey et al. 2006). Our results imply that clusters possessing significant $\mathrm{BH}$ populations are, for most of their lives, low-density objects in which the timescale for close encounters between stars and BHs is very long. It is therefore unsurprising that no BH X-ray binaries are seen in the $\sim 150$ Galactic globulars.

\section{Acknowledgements}

ADM is supported by a Marie Curie Excellence Grant from the European Commission under contract MCEXT-CT-2005-025869. MIW acknowledges support from a Royal Society University Research Fellowship. MBD is a Royal Swedish Academy Research Fellow supported by a grant from the Knut and Alice Wallenberg Foundation.

\section{References}

Aarseth S. J. 2003, Gravitational N-body Simulations. Cambridge University Press, Cambridge Elson R., Fall S. M., \& Freeman K. C. 1987, ApJ 323, 54

Elson R., Freeman K. C., \& Lauer T. R. 1989, ApJ 347, L69

Kroupa P. 2001, MNRAS 322, 231

Kulkarni S. R., Hut P., \& McMillan S. 1993, Nature 364, 421

Mackey A. D. \& Gilmore G. F. 2003a, MNRAS 338, 85

Mackey A. D. \& Gilmore G. F. 2003b, MNRAS 338, 120

Mackey A. D., et al. 2006, ApJ 653, L105

Mackey A. D., Wilkinson M. I., Davies M. B., \& Gilmore G. F. 2007a, MNRAS 379, L40

Makino J., Fukushige T., Koga M., \& Namura K. 2003, PASJ 55, 1163

Merritt D., Piatek S., Portegies Zwart S., \& Hemsendorf M. 2004, ApJ 608, L25

Sigurdsson S. \& Hernquist L. 1993, Nature 364, 423

Wilkinson M. I., Hurley J. R., Mackey A. D., Gilmore G. F., \& Tout C. A. 2003, MNRAS 343, 1025 